\title{
Diabetes Mellitus and Dyslipidemia- A Detailed Analysis
}

\author{
Brijesh Mukherjee', Monalisa Mohanty ${ }^{2}$ \\ ${ }^{1}$ Associate Professor, Department of Biochemistry, Hi-tech Medical College and Hospital, Rourkela, Odisha, ${ }^{2}$ Assistant \\ Professor, Department of Medicine, Hi-tech Medical College and Hospital, Rourkela, Odisha
}

Background: Diabetic atherosclerosis is a heterogeneous condition, and that dyslipidemia is only one aspect of the pathophysiology. However, dyslipidemia is the most readily measured and, at the present time, the most readily treated aspect of the problem. The occurrence of dyslipidemia can be reduced by good glycemic control. However poor awareness and other socio-economic factors is a major concern and pose a major hindrance in controlling blood sugar levels. Aims and Objective: This study was conducted to study the dyslipidemia pattern in diabetic patients and its association with other factors. Materials and Methods: The study included 500 subjects and was divided into two groups: Group 1(Diabetic) included 276 patients and Group 2 (Non-diabetic) included 224 patients. The study patients were screened using a pretested structured questionnaire. Fasting plasma glucose and serum lipid profile (cholesterol, TG, HDL) were estimated in automated analyzer (Erba Manheim EM 200). Results: In the current study, all characteristics of diabetes were correlated with each parameter and it was found that disproportionate body mass index, high waist girth was correlated with incidence of dyslipidemia. The education levels and financial background of the subjects were also studied and it was found to correlate well with diabetic dyslipidemia. Conclusion: Early diagnosis and treatment of dyslipidemia in diabetic patients can reduce the morbidity and mortality due to the disease.

Key words: Dyslipidemia; diabetes; mortality; morbidity
Website:

http://nepjol.info/index.php/AJMS

DOI: 10.3126/ajms.v12i5.33763

E-ISSN: 2091-0576

P-ISSN: 2467-9100

Copyright (c) 2021 Asian Journal of Medical Sciences

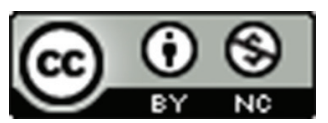

This work is licensed under a Creative Commons Attribution-NonCommercial 4.0 International License.

\section{INTRODUCTION}

As per the ICMR INDIAB study (2011), an approximate of 62.4 million people with diabetes and 77.2 million individuals with pre-diabetes are diagnosed in India. With a large population of diabetes patients, India ranks next to China in number of diabetes patients worldwide, which is estimated by World Health Organization (WHO) and International Diabetics Federation. Every 3 out of 4 diabetics suffer from dyslipidaemia. ${ }^{1}$ In diabetic patients with dyslipidaemia, 3-4 times heightened cardiovascular (CV) risk is noticed as compared to dyslipidaemia without diabetes. $^{2}$

Patients with diabetes have a 2- to 3-fold higher rate of coronary artery disease (CAD), a 4-fold higher risk of dying during acute myocardial infarction (MI), and 2-fold higher risk of post-MI morbidity than nondiabetic persons. Dr. Edwin Bierman, a leader in the field of diabetic atherosclerosis, reminded us that this is a heterogeneous condition, and that dyslipidemia is only one aspect of the pathophysiology. However, dyslipidemia is the most readily measured and, at the present time, the most readily treated aspect of the problem.

Dr. Ira J. Goldberg, ${ }^{3}$ of Columbia University, New York, discussed the pathophysiologic basis of the common form of diabetic dyslipidemia from the standpoint of the impact of hypertriglyceridemia. As shown in such studies as the United Kingdom Prospective Diabetic Study (UKPDS), 4 key features of diabetic dyslipidemia are: 1) hypertriglyceridemia, 2) a high proportion of small dense low-density lipoprotein-cholesterol (LDL), 3) low highdensity lipoprotein-cholesterol (HDL), and 4) postprandial 
lipemia. Plasma LDL levels per se are not usually higher than those of nondiabetic patients.

A cascade of pathogenic steps, resulting from insulin resistance together with dysfunction of the enzyme lipoprotein lipase (LPL), could account for most of these abnormalities. Insulin resistance in adipocytes allows exuberant lipolysis stimulated by hormone-sensitive lipase, resulting in excessive free fatty acid (FFA) release into the blood. The excess delivery of FFA to the liver (together with hepatic insulin resistance) results in upregulation of apolipoprotein B (apoB), by preventing its degradation. Therefore, the liver produces and exports an increased amount of triglyceride ( $\mathrm{TG}$ )-rich/apoB-rich very lowdensity lipoproteins (VLDL). Normally, VLDL would interact with LPL in the vessel wall of adipocyte and muscle cells, and LPL would "clear" TGs for storage in adipocytes and convert VLDL to LDL. As a result of LPL activity, HDL would also be increased.

In diabetes, LPL activity is blunted, which accounts in part for the elevated plasma VLDL-TG and the diminished HDL as well as the relatively low LDL. A second factor contributing to these circulating abnormalities is the circulating plasma enzyme cholesterol ester transfer protein (CETP). When plasma VLDL-TG is elevated, CETP causes TG to move to HDL and LDL, and, conversely, causes cholesterol ester to move from HDL and LDL to VLDL-TG. Hence, diabetics with high plasma VLDL concomitantly have low measured plasma HDL because the cholesterol ester is being transferred out of the HDL fraction, as well as a diminution in the size of the LDL particle because LDL becomes TG enriched, and this is converted by hepatic lipase to small, dense LDL. This explains, to a large extent, 3 of the 4 dyslipidaemic features.

The fourth feature is postprandial lipemia. Dietary fat is absorbed in the form of chylomicrons, and, in diabetic patients, the defect in LPL leads to a persistent and prolonged elevation of chylomicron-TG in the blood. Furthermore, there is a second defect in the normal processing of "chylomicron remnant" particles, the product of LPL-mediated lipolysis of chylomicrons. Chylomicron remnants are normally cleared by a special pathway in the liver via attachment to specific heparan sulfate proteoglycans. The proteoglycan trapping mechanism is known to be deficient in diabetes (at least, in diabetic mice); hence, people with diabetes may accumulate chylomicron remnants in the blood as well.

\section{Aims and Objective}

The objective of the study was to assess the prevalence of dyslipidaemia as a cardiovascular risk factor amongst patients with diabetes in and around Rourkela.

\section{MATERIALS AND METHODS}

The study was conducted at Hi-tech Medical College and Hospital, Rourkela between January to December, 2019. All the subjects were asked to sign the informed consent after registration. The study was approved by the Institutional Ethics Committee.

This study included total 500 patients, who were assigned to two separate groups: Group 1 (Diabetic) included 276 patients and Group 2 (Non-diabetic) included 224 patients. Patients aged between 18-65 years were included in the study.

The study patients were screened using a pretested structured questionnaire. Data with respect to sociodemographic characteristics, age, sex, socioeconomic status, per capita monthly income, family size, duration of diabetes, comorbidity, history of cardiovascular disease, family history of diabetes, dietary habits, tobacco and alcohol use, physical activity etc., were collected. Height, weight, and waist circumference were measured, and BMI was calculated for all of them. Pulse and BP were measured by manual sphygmomanometer in sitting position.

Fasting plasma glucose and serum lipid profile (cholesterol, TG, HDL) were estimated immediately after in automated analyzer (Erba Manheim EM 200) and HbA1c in D10 hematoanalyzer. The LDL was calculated by Friedewald'smethod.

\section{RESULTS}

A total of 500 patients were involved in this study, who were assigned into two groups: Group 1 (Diabetic) included 276 patients and Group 2 (Non-diabetic) included 224 patients. The male to female ratio in both groups are shown in Figure 1.

Amongst the diabetic group (Group 1) 58.33\% patients had raised triglycerides (TG) values (i.e. $\geq 150 \mathrm{mg} / \mathrm{dl}$ ), $47.82 \%$ patients had raised VLDL values (i.e. $>30 \mathrm{mg} / \mathrm{dl}$ ), $36.96 \%$ patients had lower HDL values (i.e. $<40 \mathrm{mg} / \mathrm{dl}$ ), $31.52 \%$ patients had raised total cholesterol values (i.e. $\geq$ $200 \mathrm{mg} / \mathrm{dl}$ ) and $25.36 \%$ patients had raised LDL values (i.e. $\geq 130 \mathrm{mg} / \mathrm{dl}$ ). In diabetes group $83.33 \%$ patients had dyslipidaemia and $16.67 \%$ patients had normal lipid profile (Figure 2). In this group, maximum patients diagnosed had elevated TG values when compared to other parameters of dyslipidaemia.

However, in Non-diabetes group (Group 2) 46.43\% patients had lower values of HDL $25.45 \%$ patients had 


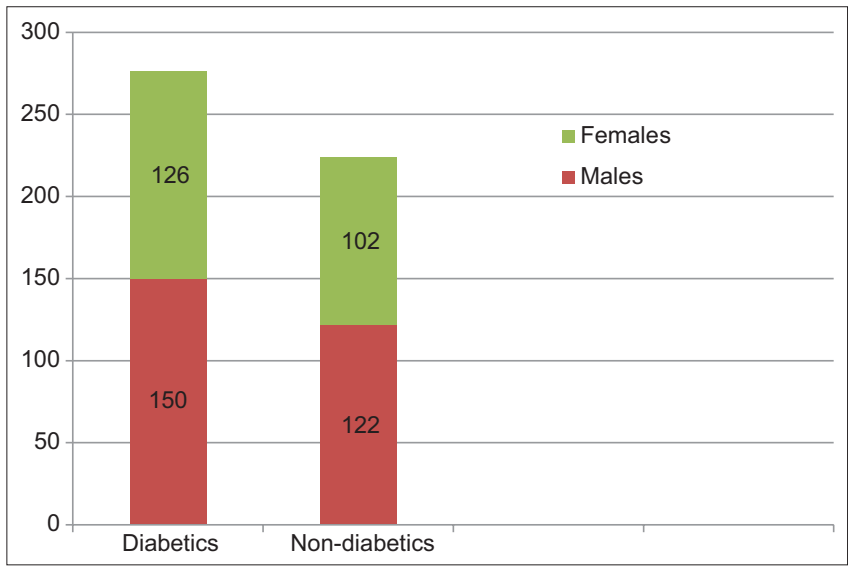

Figure 1: Male: female ratio in each group

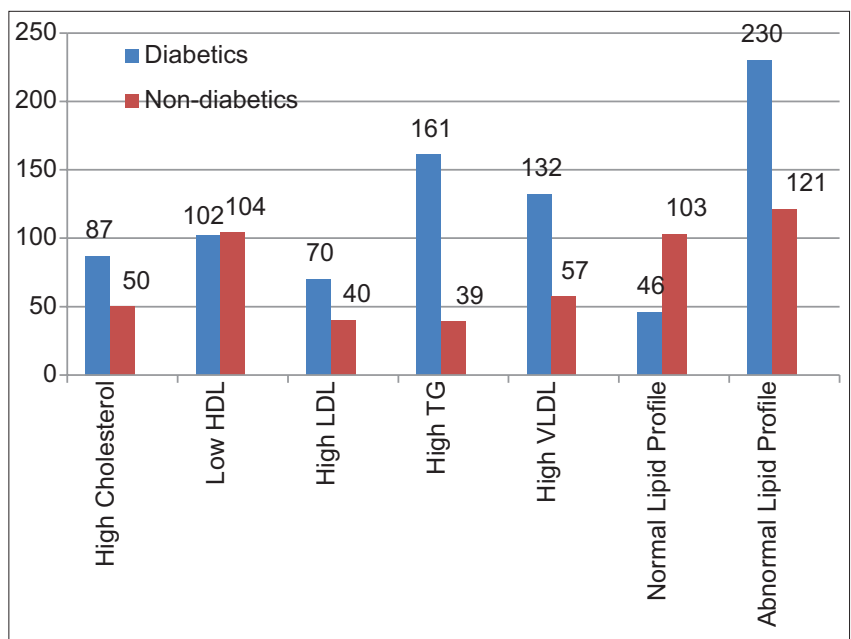

Figure 2: Lipid profile distribution among diabetic and non-diabetic group

raised Very Low-Density Lipoprotein (VLDL) values, $22.32 \%$ patients had raised cholesterol values, $17.86 \%$ patients had raised LDL values and $17.41 \%$ patients had raised TG values. In Non-diabetes group 54.01\% patients had dyslipidaemia and $45.99 \%$ patients had normal lipid profile (Figure 2). In this group, maximum patients were identified with abnormal HDL values followed by VLDL.

The prevalence of diabetes in different age group is shown in Figure 3. It was observed that prevalence increases with increasing age. $56.16 \%$ of the diabetic subjects were above 50 years of age.

Obesity is a known lifestyle disease and is correlated with diabetes and dyslipidemia. Waist circumference and body mass index (BMI) are often used to define obesity. It was found that diabetic group had a greater number of obese patients compared to the non-diabetic population (Figure 4). The obesity was assessed based on the BMI measurement. Also, waist circumference one of the parameters to assess obesity was considered during the

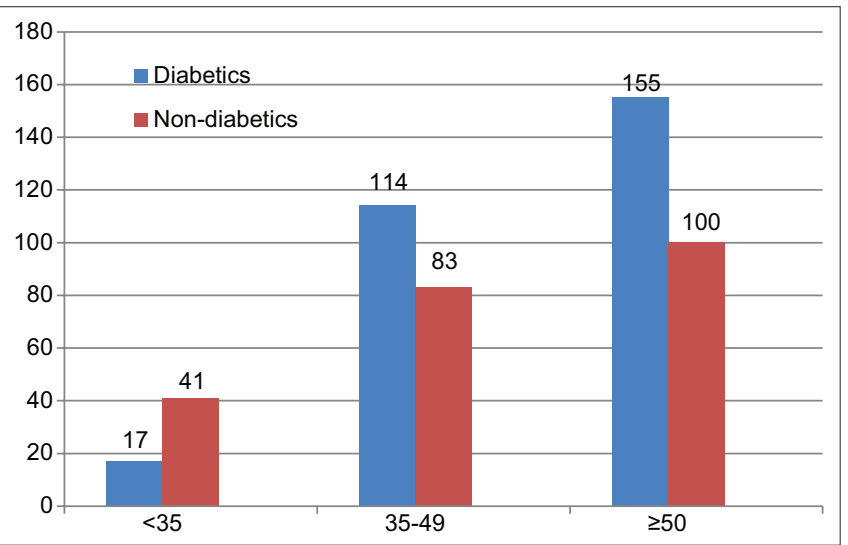

Figure 3: Diabetes prevalence versus non-diabetic age comparison

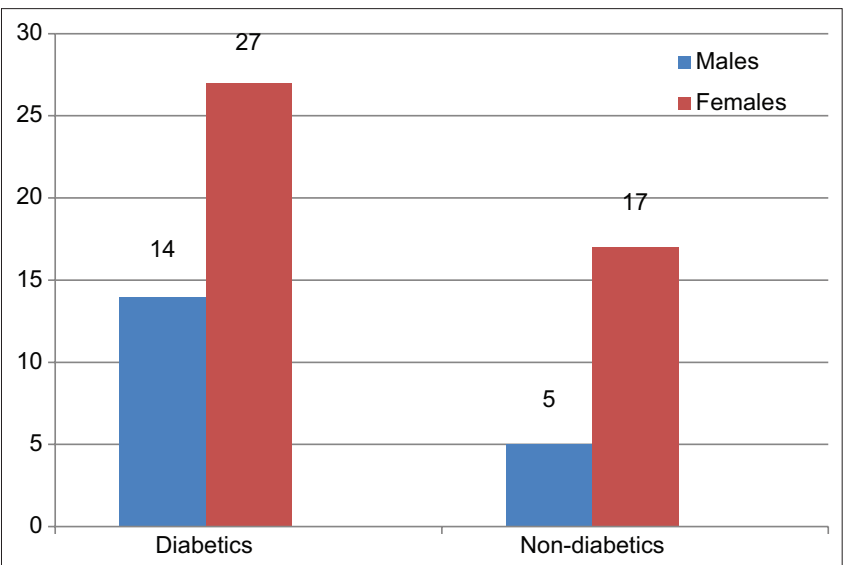

Figure 4: Comparison of BMI ( $\left.\geq 30 \mathrm{~kg} / \mathrm{m}^{2}\right)$ in both Diabetic and Nondiabetic group

study, wherein female patients outnumbered the male population in obesity in both diabetic and non-diabetic group (Figure 5).

It was observed that $38.46 \%$ of the diabetic population had higher level of education, which on further assessment proved that changing lifestyle in the educated population is one of the major contributory factors for dyslipidaemia, CVD and other risk factors (Figure 6).

Individuals with family history of diabetes and hypertension in either of the parents or both the parents should take necessary steps as they are more prone to become hypertensive or diabetic as age progresses (Figure7 and 8).

Figure 9 shows that $61.23 \%$ hypertensive patients and $92.75 \%$ diabetic patients were on medication in the Diabetic group, while $14.29 \%$ hypertensive patients were on medication in the Non-diabetic group.

Figure 10 depicts the number of subjects belonging to different income groups. More than $50 \%$ of diabetic subjects' monthly income exceeded INR 20000. 


\section{DISCUSSION}

Diabetes is becoming a disease of global concern because of its high escalating numbers. The number of diabetics is expected to increase from 382 million in 2013 to 592 million by $2035 .{ }^{4}$ The leading cause of morbidity and mortality for patients with type 2 diabetes is CVD. According to American Heart Association, at least $68 \%$ of the people with diabetes aged 65 or older die of heart disease and $16 \%$ die of stroke. Furthermore, adults with diabetes are two to four times more likely to have CVD than adults without diabetes. Thus, it is considered as coronary artery disease equivalent. ${ }^{5}$ The overall cardiometabolic risk is driven by a complex interplay between several non-modifiable (age, gender, genetics), modifiable (hypertension, hyperlipidemia, hyperglycemia) factors and the components of the metabolic syndrome commonly associated with type 2 diabetes. $^{5}$

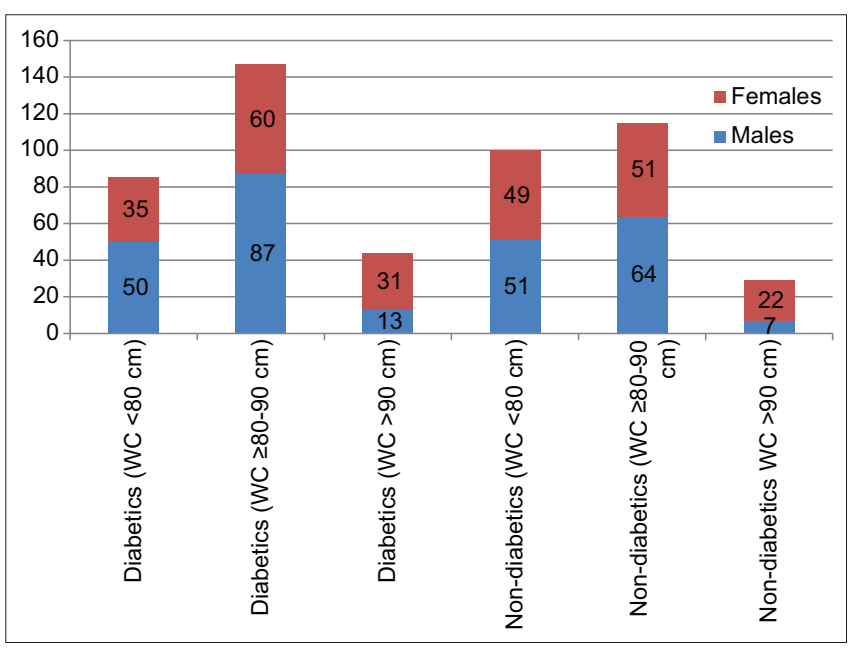

Figure 5: Waist circumference comparison between both sexes
We found out higher levels of TG, VLDL and LDL in diabetic patients compared to normal subjects. Similar findings were observed by Agarwal et al. and Behera SN et al. ${ }^{6,7}$

Lifestyle modifications are the first-line intervention in the management of diabetes dyslipidemia, and include weight loss, dietary modification, and aerobic exercise. ${ }^{8}$ Obesity increases insulin resistance and is associated with increased triglycerides and LDL cholesterol and decreased HDL cholesterol. ${ }^{9}$ Weight loss is known to be associated with improvements in lipids and other cardiovascular risk factors including the incidence of type 2 diabetes, ${ }^{10,11}$ and should therefore be encouraged in overweight patients with diabetes. We found out there is obvious link between obesity and lifestyle with diabetes and dyslipidemia. Hence early lifestyle modification and weight control is of immense importance.

In the current study, all characteristics of diabetes were correlated with each parameter and it was found that disproportionate body mass index, high waist girth were correlated with incidence of CVD. Literacy is one of the factors found intermediate responsible for CVD. The more educated, the more advances in current lifestyle are observed whereas due to illiteracy, there is lesser health awareness, and both together result in an increased incidence of CVD. This again is in agreement to similar earlier studies. ${ }^{8}$

Various hypotheses have been put forward to explain the typical diabetic dyslipidemia, summary of evidences point out that the overproduction of VLDL particles initiates a sequence of events that results in the atherogenic lipid triad consisting of elevated plasma concentrations of

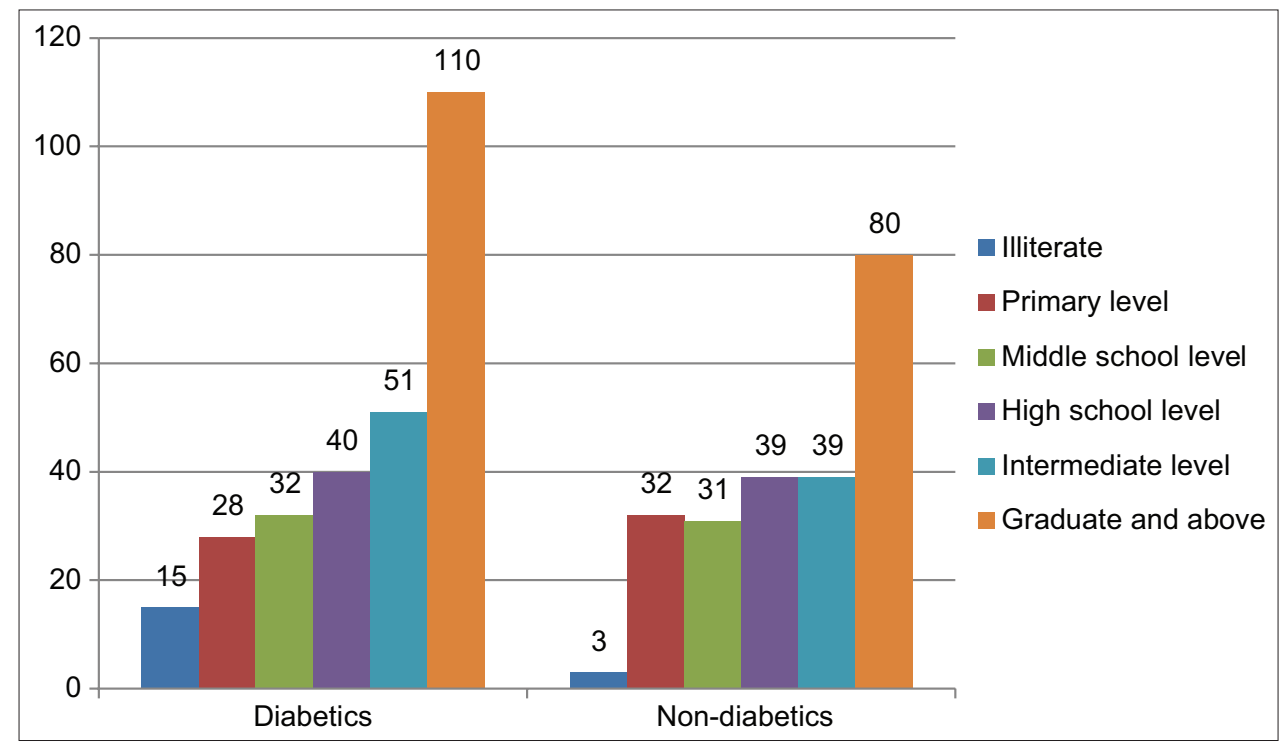

Figure 6: Literacy comparison among both groups 




Figure 7: Comparison of both groups with history of diabetes

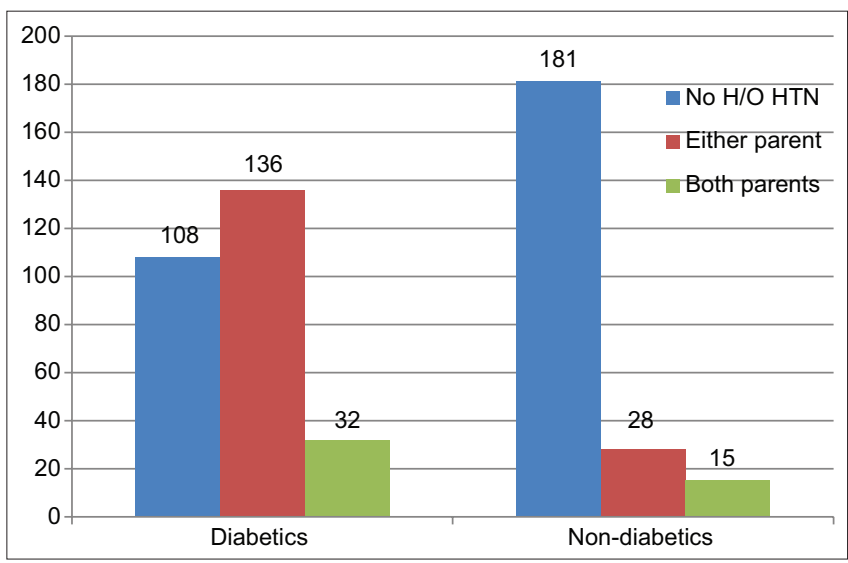

Figure 8: Comparison of both groups with history of hypertension

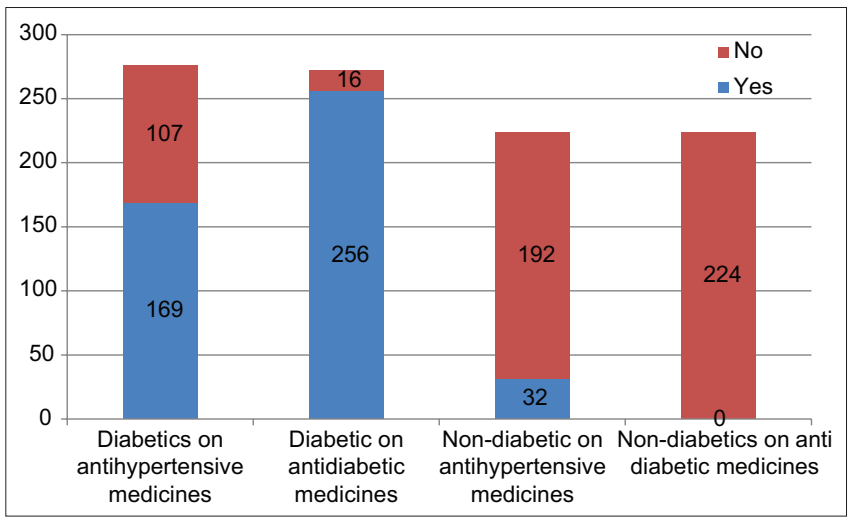

Figure 9: Comparison of medication among both groups

fasting and post-prandial TRLs, small dense LDL and low HDL cholesterol. However, we could not find enough differences between HDL levels in the two groups. However, VLDL and TG levels were markedly increased in diabetic patients. Both TG and VLDL are important markers for risk of CVD and should be screened in all diabetic patients.



Figure 10: Income distribution among the diabetic and non-diabetic groups

\section{CONCLUSION}

More cardiovascular disease occurs in patients with either type 1 or 2 diabetes. The link between diabetes and atherosclerosis is, however, not completely understood. Among the metabolic abnormalities that commonly accompany diabetes are disturbances in the production and clearance of plasma lipoproteins. Moreover, development of dyslipidemia may be a harbinger of future diabetes. A recent position statement from the ADA recommends a screening lipid profile at the time of diagnosis of diabetes, at age 40 years, and periodically thereafter. Strict recommendations for lifestyle modification and optimization of glycemic control can decrease the burden of diabetic dyslipidemia.

\section{REFERENCES}

1. Selby JV, Peng T, Karter AJ, Alexander M, Sidney S, Lian J, et al. High rates of concurrence of hypertension, elevated low-density lipoprotein cholesterol, and diabetes mellitus in a large managed care population. J Americ Man care. 2004;10(2):163-170.

2. Stamler J, Vaccaro O, Neaton JD, Wentworth D, Multiple Risk Factor Intervention Trial Research Group. Diabetes, other risk factors, and 12-yr cardiovascular mortality for men screened in the Multiple Risk Factor Intervention Trial. Diabetes care. 1993;16(2):434-44.

https://doi.org/10.2337/diacare.16.2.434

3. Goldberg IJ. Diabetic Dyslipidemia. Statins Versus Fibrates in the Treatment of Diabetic Dyslipidemia. Symposium.Program and abstracts of the 61st Scientific Sessions of the American Diabetes Association; June 22-26, 2001; Philadelphia, Pennsylvania.

4. Guariguata L, Whiting DR, Hambleton I, Beagley J, Linnenkamp $U$ and Shaw JE. Global estimates of diabetes prevalence for 2013 and projections for 2035. Diabetes Res Clin Pract. 2014;103(2):137-149.

https://doi.org/10.1016/j.diabres.2013.11.002

5. Risk factors for coronary artery disease in non-insulin dependent diabetes mellitus: United Kingdom Prospective Diabetes Study (UKPDS: 23). BMJ. 1998;316(7134):823-828.

https://doi.org/10.1136/bmj.316.7134.823

6. Agarwal AK, Singla S, Singla S, Singla R, Lal A, Wardhan H, et al. Prevalence of coronary risk factors in type 2 diabetics 
without manifestations of overt coronary heart disease. J Assoc Physicians India. 2009; 57:135-142.

7. Behera SN and Patro S. Dyslipidaemia pattern amongst diabetic patients visiting a tertiary care hospital in Eastern Odisha. Int $\mathrm{J}$ Adv Med. 2017;4(6):1662-1667.

https://doi.org/10.18203/2349-3933.ijam20175186

8. Warraich HJ, Wong ND and Rana JS. Role for combination therapy in diabetic dyslipidemia. Curr Cardiol Rep. 2015;17:32. https://doi.org/10.1007/s11886-015-0589-5

9. Durrington PN. Hyperlipidaemia: diagnosis and management. London: Hodder Arnold; 2007. https://doi.org/10.1201/b13464
10. Wood PD, Stefanick ML, Dreon DM, Frey-Hewitt B, Garay SC, Williams PT, et al. Changes in plasma lipids and lipoproteins in overweight men during weight loss through dieting as compared with exercise. N Engl J Med. 1988; 319:1173-1179.

https://doi.org/10.1056/NEJM198811033191801

11. Rock CL, Flatt SW, Pakiz B, Taylor KS, Leone AF, Brelje K, et al. Weight loss, glycemic control, and cardiovascular disease risk factors in response to differential diet composition in a weight loss program in type 2 diabetes: a randomized controlled trial. Diabetes Care. 2014; 37:1573-1580

https://doi.org/10.2337/dc13-2900

Author's contribution:

BM - Concept and design of the study, prepared first draft of manuscript, Interpreted the results, reviewed the literature and manuscript preparation;

MM - Concept, coordination, review of literature and manuscript preparation, statistically analysed and interpreted, preparation of manuscript and revision of the

manuscript.

Work Attributed to:

Department of Biochemistry, Hi-tech Medical College and Hospital, Rourkela- 769004, Odisha, India.

Orcid ID:

Dr. Brijesh Mukherjee - (1) https://orcid.org/0000-0003-2038-127X

Source of Funding: None, Conflict of Interest: None. 